Research Paper

\title{
High Expression of $17 \beta$-hydroxysteroid Dehydrogenase Type 2 is Associated with a Better Prognosis in Urothelial Carcinoma of the Urinary Tract
}

\author{
Chieh-Tien Wang ${ }^{1,2}$, Chien-Feng $\mathrm{Li}^{33,4,5,6}$, Wen-Jeng $\mathrm{Wu}^{7,8,9}$, Chun-Nung Huang7,8, Ching-Chia Li ${ }^{7,8,9}$, \\ Wei-Ming Li ${ }^{7,8}$, Ti-Chun Chan ${ }^{3}$, Peir-In Liang10, Chung-Hsi Hsing11, Kuang-Ming Liao ${ }^{12}$ \\ 1. Department of Pathology, Chi Mei Medical Center, Liouying, Taiwan; \\ 2. Department of Medical Laboratory Science and Biotechnology, Chung Hwa University of Medical Technology; \\ 3. Department of Pathology, Chi Mei Medical Center, Tainan, Taiwan; \\ 4. National Institute of Cancer Research, National Health Research Institutes, Tainan, Taiwan; \\ 5. Department of Biotechnology, Southern Taiwan University of Science and Technology, Tainan, Taiwan; \\ 6. Institute of Clinical Medicine, Kaohsiung Medical University, Kaohsiung, Taiwan; \\ 7. Department of Urology, Faculty of Medicine, Kaohsiung Medical University; \\ 8. Department of Urology, Kaohsiung Medical University Hospital, Kaohsiung Medical University; \\ 9. Department of Urology, Kaohsiung Municipal Ta-Tung Hospital; \\ 10. Department of Pathology, Kaohsiung Medical University Hospital, Kaohsiung Medical University; \\ 11. Department of Anesthesiology, Chi Mei Medical Center, Tainan, Taiwan; \\ 12. Department of Internal Medicine, Chi Mei Medical Center, Chiali, Taiwan. \\ $\square$ Corresponding author: Kuang-Ming Liao, Department of Internal Medicine, Chi Mei Medical Center, Chiali, Taiwan. No.606, Jialixing, Jiali District, Tainan \\ City 722, Taiwan (E-mail: abc8870@yahoo.com.tw).
}

() Ivyspring International Publisher. Reproduction is permitted for personal, noncommercial use, provided that the article is in whole, unmodified, and properly cited. See http://ivyspring.com/terms for terms and conditions.

Received: 2016.07.08; Accepted: 2016.09.01; Published: 2016.10.26

\begin{abstract}
Object: To identify associations between 17 $\beta$-hydroxysteroid dehydrogenase type 2 (HSD17B2) expression and clinicopathological variables and prognoses in patients with urothelial carcinoma of the urinary tract.

Materials and Methods: We analyzed one public transcriptome dataset (GSE31684) from the Gene Expression Omnibus. HSD17B2 showed the highest log2-transformed fold-change in expression, and it was therefore further analyzed. We enrolled 340 upper urinary tract and 295 urinary bladder tissue samples that were obtained from patients with urothelial carcinoma between 1996 and 2004 to evaluate the expression of HSD17B2 using immunohistochemistry. The endpoints were disease-specific survival and metastasis-free survival. Univariate and multivariate analyses were performed to assess the relationships between HSD17B2, survival and clinicopathological parameters.

Results: High expression of HSD17B2 was significantly associated with better clinicopathological parameters, including the following parameters in urothelial carcinoma of upper urinary tracts: $\mathrm{Ta}$ (non-invasive papillary carcinoma) and Tl (invaded subepithelial connective tissue) disease, without nodal metastasis, without vascular invasion, without perineal invasion and with a low histological grade, and the following parameters specifically in urothelial carcinoma of the urinary bladder: Ta (noninvasive papillary carcinoma) and Tl (invaded into the lamina propria but not into the muscularis propria) disease, without nodal metastasis (all $\mathrm{P}<0.05$ ). Additionally, HSD17B2 high expression predicted a better prognosis, including improved disease-specific survival and metastasis-free survival in urothelial carcinomas of the urinary tract system.

Conclusions: High expression of HSD17B2 is associated with a better prognosis and is therefore a prognostic biomarker that can be used to predict favorable survival in patients with urothelial carcinoma of the urinary tract system.
\end{abstract}

Key words: $17 \beta$-hydroxysteroid dehydrogenase type 2, Urothelial carcinoma, Prognosis. 


\section{Introduction}

The enzyme $17 \beta$-hydroxysteroid dehydrogenase type 2 (HSD17B2) plays a vital role in steroid metabolism, including the conversion of the biologically potent factor estradiol into the weakly estrogenic estrone in addition to testosterone and androstenedione [1]. There are 14 types of $17 \beta$-hydroxysteroid dehydrogenases, and they are named based on their functions, which include the activation of the reduction or oxidation of 17-keto and 17-hydroxy steroids. These processes involve $\mathrm{NAD}+/ \mathrm{NADH}$ or NADP+/NADPH-dependent biological reactions [2]. Human HSD17B2 is a protein that consists of 387 amino acids and has a molecular weight of 42,782 Da. From an analysis of its amino acid sequence motifs, HSD17B2 was found to be associated with the membranes of the endoplasmic reticulum [3].

Previous studies have shown that HSD17B2 is associated with human anterior pituitary adenomas [4], that a low level of expression of HSD17B2 is associated with a poor prognosis in breast cancer [5], and that HSD17B2 is associated with an increased risk of sporadic and familial prostate cancer [6]. To our knowledge, no previous studies have investigated the relationship between urothelial carcinoma of the urinary tract system and HSD17B2. The aim of this study was to investigate the association between HSD17B2 and prognosis in urothelial carcinoma of the urinary tract system.

\section{Materials and Methods}

\section{Analysis of published transcriptomic datasets}

We first analyzed one public transcriptome dataset (GSE31684) that was obtained from the NIH Gene Expression Omnibus. For the data analysis, the raw CEL files available in the Affymetrix HUMAN Genome U133 Plus 2.0 microarray platform were imported into Nexus Expression 3 software (BioDiscovery) to analyze all probe sets without using pre-selection or filtering, as previously described [7-10]. Supervised comparative analyses and functional profiling were performed to identify significantly differentially expressed genes, and special attention was paid to pathways known to be involved in lipid biosynthetic process in Gene Ontology (GO:0008610). Those genes with $P<0.01$ and log2-transformed expression fold change $> \pm 0.1$ were chosen and the gene with most significant $P$ value and the highest log2-transformed fold-change in expression was selected for further validation. To cross-validate the findings, the transcriptomic data from another dataset (GSE32894), which was generated using an Illumina HumanHT-12 V3.0 expression bead chip, was also analyzed.

\section{Patients and tumor specimens}

This study was approved by the Institutional Review Board of the Chi Mei Medical Center (IRB10501005). We enrolled 340 upper urinary tract and 295 urinary bladder formalin-fixed and paraffin-embedded tissue samples that were obtained from patients with urothelial carcinoma between 1996 and 2004. These samples were used to evaluate HSD17B2 using immunohistochemistry. Patients with urothelial carcinoma of the urinary tract received biopsies in the Chi-Mei Medical Center and subsequently underwent a regular treatment and follow-up schedule in the hospital. All patients with urothelial carcinoma of the upper urinary tract received a nephron-ureterectomy, including the resection of the bladder cuff, and a regional lymphadectomy. Patients with superficial urothelial carcinoma of the urinary bladder (pTa and pT1) underwent a transurethral resection of the bladder tumor either with or without the intravesicular application of the bacillus Calmette-Guérin vaccine. For patients with tumor recurrence, a salvage cystectomy was performed.

Radical cystectomy with bilateral pelvic iliac lymphadenectomy was performed for tumors that had invaded the muscle layer of the bladder. Patients with urothelial carcinoma of the urinary bladder with either pT3-4 or node-positive disease received cisplatin-based chemotherapy. Only 29 of 106 patients had a pathologic stage of T3 or higher with/without lymph node metastasis, and these patients received adjuvant chemotherapy for upper tract urothelial malignancies. No patients received neoadjuvant chemotherapy in our study. TNM staging was determined using the most invasive lesion at any multifocality, and we excluded patients with combined urothelial carcinoma of both the upper tract and the urinary bladder.

The mean follow-up durations for patients with upper tract and urinary bladder carcinoma were 44.7 and 30.8 months, respectively. Vascular invasion was defined as the presence of tumor cells inside the endothelium-lined vascular channel within the tumor and surrounding tissues. Perineural invasion was used to describe the spread of cancer into the space surrounding a nerve. The patients were classified into 2 groups; a low mitotic activity group in which the mitotic rate less than 10 per 10 high-power fields and a high mitotic activity group in which the mitotic rate was 10 or more per 10 high-power fields. These methods have also been described in our previous studies [7-10]. 
Tissues obtained from patients with an initial diagnosis of urothelial carcinoma of the urinary tract with and without metastasis after biopsy were reviewed by two pathologists (C.F. Li and P.I. Liang). The histological classification and stage of each tumor was determined based on hematoxylin- and eosin-stained sections according to the classification system for malignant tumors of the American Joint Committee on Cancer Tumor-Nodes-Metastasis (TNM) [11].

\section{Immunohistochemical staining and assessment of HSD17B2}

The tissues, which were previously fixed in formalin and embedded in paraffin, were cut into 4 $\mu \mathrm{m}$-thick sections. To remove the paraffin wax, the sections were placed in vessels containing fresh xylene, rehydrated in ethanol, and then heated in a microwave. These methods were described in our previous study [7-10]. The tissues were then washed with TRIS buffer and incubated with anti-HSD17B2 monoclonal antibodies (1:100; Abcam).

\section{Statistical analysis}

We used the Statistical Package for the Social Sciences (SPSS) version 14 for data analysis. Chi-square tests were used to analyze categorical variables, and Fisher's exact test was used to analyze contingency variables. These tests were used to determine whether there was a significant association between HSD17B2 expression in urothelial carcinoma tissues and various clinicopathological parameters, including primary tumor $(\mathrm{T})$, nodal metastasis, histological grade, vascular invasion, perineural invasion, and mitotic rate (per 10 high power fields). The endpoints were disease-specific survival and metastatic-free survival. The endpoint results were calculated from the time of diagnosis with urothelial carcinoma to the date of an event (e.g., death or metastasis). Univariate and multivariate analyses were performed to assess the relationships between disease-specific survival or metastatic-free survival and clinicopathological parameters.

Kaplan-Meier curves and estimates of survival data were subsequently used to evaluate the relationship between HSD17B2 expression and survival times. To compare survival times between groups, we used log rank tests to determine whether the survival curves were identical. A P-value of less than 0.05 was considered significant.

\section{Results}

\section{Lipid biosynthesis and urothelial carcinoma}

Table 1 includes a summary of genes that were differentially expressed in the transcriptome of urothelial carcinoma of the urinary bladder (GSE31684) that are known to be involved in lipid biosynthetic process. These markers were positively associated with cancer invasiveness in urothelial carcinoma of the urinary bladder. Among the genes identified in this analysis, HSD17B2 was the most significantly associated, in that its expression was lower in T2-4 than in Ta-T1 lesions and had a log2 ratio during progression between these stages of -3.1569 (Figure $\mathbf{1}$ and Table 1, $P<0.0001$ ). Additionally, we also tested the expression of HSD17B2 in another GEO dataset (GSE32894) and found that HSD17B2 expression also decreased as urothelial carcinoma progressed (Figure S1 and Table S1). Based on these data, we used immunohistochemistry to analyze the association between HSD17B2 and clinicopathological characteristics in patients with urothelial carcinoma of the urinary system.

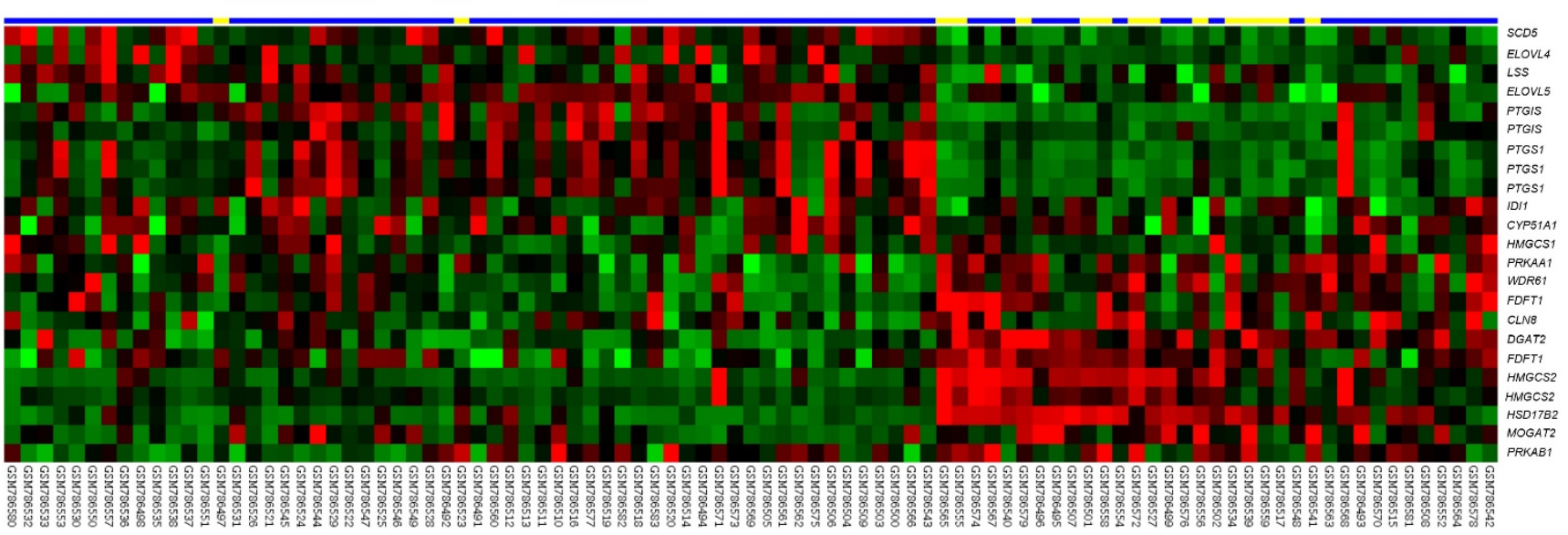

Figure 1. An analysis of gene expression in urothelial carcinoma of the urinary tract system was performed using a published transcriptomic dataset (GSE31684). The clustering analysis focused on genes involved in regulating steroid metabolism. The results revealed that $17 \beta$-hydroxysteroid dehydrogenase type 2 was the most significantly down-regulated gene and that its expression was associated with a decrease in pT status. The PT statuses of the tissue specimens obtained from different tumors are shown on top of the heat map, and the upregulation or downregulation of the genes is illustrated as a spectrum of red and green brightness, respectively. Unaltered genes are shown as black. 
Table 1. Summary of differentially expressed genes associated with lipid biosynthetic process (GO:0008610) that showed positive associations with cancer invasiveness in the transcriptome of urothelial carcinoma of the urinary bladder (GSE3 1684).

\begin{tabular}{|c|c|c|c|c|c|}
\hline \multirow[t]{2}{*}{ Probe } & \multicolumn{2}{|c|}{$\begin{array}{l}\text { Comparing T2-4 to } \\
\text { Ta-T1 }\end{array}$} & \multirow[t]{2}{*}{$\begin{array}{l}\text { Gene } \\
\text { Symbol }\end{array}$} & \multirow[t]{2}{*}{ Gene Title } & \multirow[t]{2}{*}{ Molecular Function } \\
\hline & Log2 ratio & $P$-value & & & \\
\hline 204818_at & -3.1569 & $<0.0001$ & HSD17B2 & $\begin{array}{l}\text { hydroxysteroid (17-beta) } \\
\text { dehydrogenase } 2\end{array}$ & estradiol 17-beta-dehydrogenase activity, oxidoreductase activity \\
\hline 204607_at & -2.4778 & $<0.0001$ & HMGCS2 & $\begin{array}{l}\text { 3-hydroxy-3-methylglutaryl-Coenzyme } \\
\text { A synthase } 2 \text { (mitochondrial) }\end{array}$ & hydroxymethylglutaryl-CoA synthase activity, transferase activity \\
\hline 226064_s_at & -1.0777 & $<0.0001$ & $D G A T 2$ & $\begin{array}{l}\text { diacylglycerol O-acyltransferase } \\
\text { homolog } 2 \text { (mouse) }\end{array}$ & $\begin{array}{l}\text { acyltransferase activity, diacylglycerol O-acyltransferase activity, } \\
\text { transferase activity }\end{array}$ \\
\hline 241954_at & -0.8037 & 0.0010 & FDFT1 & $\begin{array}{l}\text { Farnesyl-diphosphate } \\
\text { farnesyltransferase } 1\end{array}$ & $\begin{array}{l}\text { catalytic activity, farnesyl-diphosphate farnesyltransferase activity, } \\
\text { magnesium ion binding, oxidoreductase activity, protein binding, } \\
\text { transferase activity }\end{array}$ \\
\hline 214917_at & -0.6561 & 0.0070 & PRKAA1 & $\begin{array}{l}\text { protein kinase; AMP-activated; alpha } 1 \\
\text { catalytic subunit }\end{array}$ & $\begin{array}{l}\text { ATP binding, cAMP-dependent protein kinase activity, kinase } \\
\text { activity, magnesium ion binding, metal ion binding, nucleotide } \\
\text { binding, protein kinase activity, protein serine/threonine kinase } \\
\text { activity, transferase activity }\end{array}$ \\
\hline 240110_at & -0.6423 & 0.0001 & HMGCS2 & $\begin{array}{l}\text { 3-hydroxy-3-methylglutaryl-Coenzyme } \\
\text { A synthase } 2 \text { (mitochondrial) }\end{array}$ & hydroxymethylglutaryl-CoA synthase activity, transferase activity \\
\hline 208647_at & -0.5354 & 0.0097 & FDFT1 & $\begin{array}{l}\text { farnesyl-diphosphate } \\
\text { farnesyltransferase } 1\end{array}$ & $\begin{array}{l}\text { catalytic activity, farnesyl-diphosphate farnesyltransferase activity, } \\
\text { magnesium ion binding, oxidoreductase activity, protein binding, } \\
\text { transferase activity }\end{array}$ \\
\hline 201834_at & -0.5118 & $<0.0001$ & PRKAB1 & $\begin{array}{l}\text { protein kinase; AMP-activated; beta } 1 \\
\text { non-catalytic subunit }\end{array}$ & $\begin{array}{l}\text { hydrolase activity; hydrolyzing O-glycosyl compounds, protein } \\
\text { binding }\end{array}$ \\
\hline 229958_at & -0.4394 & 0.0001 & CLN8 & $\begin{array}{l}\text { ceroid-lipofuscinosis; neuronal } 8 \\
\text { (epilepsy; progressive with mental } \\
\text { retardation) }\end{array}$ & \\
\hline 237208_at & -0.4029 & 0.0022 & WDR61 & WD repeat domain 61 & $\begin{array}{l}\text { catalytic activity, isomerase activity, triose-phosphate isomerase } \\
\text { activity }\end{array}$ \\
\hline 232428_at & -0.2564 & 0.0015 & MOGAT2 & monoacylglycerol O-acyltransferase 2 & $\begin{array}{l}\text { 2-acylglycerol O-acyltransferase activity, acetyltransferase activity, } \\
\text { acyltransferase activity, transferase activity }\end{array}$ \\
\hline 208131_s_at & 1.6423 & $<0.0001$ & PTGIS & prostaglandin I2 (prostacyclin) synthase & $\begin{array}{l}\text { heme binding, iron ion binding, isomerase activity, metal ion } \\
\text { binding, monooxygenase activity, prostaglandin-I synthase activity }\end{array}$ \\
\hline 224901_at & 1.3137 & $<0.0001$ & SCD5 & stearoyl-CoA desaturase 5 & $\begin{array}{l}\text { iron ion binding, oxidoreductase activity, oxidoreductase activity; } \\
\text { acting on paired donors; with oxidation of a pair of donors resulting } \\
\text { in the reduction of molecular oxygen to two molecules of water, } \\
\text { stearoyl-CoA 9-desaturase activity }\end{array}$ \\
\hline 215813_s_at & 0.8976 & 0.0003 & PTGS1 & $\begin{array}{l}\text { prostaglandin-endoperoxide synthase } 1 \\
\text { (prostaglandin } \mathrm{G} / \mathrm{H} \text { synthase and } \\
\text { cyclooxygenase) }\end{array}$ & $\begin{array}{l}\text { iron ion binding, metal ion binding, oxidoreductase activity, } \\
\text { oxidoreductase activity; acting on single donors with incorporation } \\
\text { of molecular oxygen; incorporation of two atoms of oxygen, } \\
\text { peroxidase activity, prostaglandin-endoperoxide synthase activity }\end{array}$ \\
\hline 208881_x_at & 0.8359 & 0.0007 & IDI1 & $\begin{array}{l}\text { isopentenyl-diphosphate delta } \\
\text { isomerase } 1\end{array}$ & $\begin{array}{l}\text { hydrolase activity, isomerase activity, isopentenyl-diphosphate } \\
\text { delta-isomerase activity, magnesium ion binding, metal ion binding }\end{array}$ \\
\hline 238669_at & 0.8155 & 0.001 & PTGS1 & $\begin{array}{l}\text { prostaglandin-endoperoxide synthase } 1 \\
\text { (prostaglandin } \mathrm{G} / \mathrm{H} \text { synthase and } \\
\text { cyclooxygenase) }\end{array}$ & $\begin{array}{l}\text { iron ion binding, metal ion binding, oxidoreductase activity, } \\
\text { oxidoreductase activity; acting on single donors with incorporation } \\
\text { of molecular oxygen; incorporation of two atoms of oxygen, } \\
\text { peroxidase activity, prostaglandin-endoperoxide synthase activity }\end{array}$ \\
\hline 205128_x_at & 0.8019 & 0.0036 & PTGS1 & $\begin{array}{l}\text { prostaglandin-endoperoxide synthase } 1 \\
\text { (prostaglandin } \mathrm{G} / \mathrm{H} \text { synthase and } \\
\text { cyclooxygenase) }\end{array}$ & $\begin{array}{l}\text { iron ion binding, metal ion binding, oxidoreductase activity, } \\
\text { oxidoreductase activity; acting on single donors with incorporation } \\
\text { of molecular oxygen; incorporation of two atoms of oxygen, } \\
\text { peroxidase activity, prostaglandin-endoperoxide synthase activity }\end{array}$ \\
\hline 208788_at & 0.6671 & 0.0043 & ELOVL5 & $\begin{array}{l}\text { ELOVL family member } 5 \text {; elongation of } \\
\text { long chain fatty acids (FEN1/Elo2; } \\
\text { SUR4/Elo3-like; yeast) }\end{array}$ & \\
\hline 219532_at & 0.6276 & $<0.0001$ & ELOVL4 & $\begin{array}{l}\text { elongation of very long chain fatty acids } \\
\text { (FEN1/Elo2; SUR4/Elo3; yeast)-like } 4\end{array}$ & G-protein coupled photoreceptor activity \\
\hline 202314_at & 0.6199 & 0.0017 & CYP51A1 & $\begin{array}{l}\text { cytochrome P450; family } 51 \text {; subfamily } \\
\text { A; polypeptide } 1\end{array}$ & $\begin{array}{l}\text { heme binding, iron ion binding, metal ion binding, monooxygenase } \\
\text { activity, oxidoreductase activity, protein binding, sterol } \\
\text { 14-demethylase activity }\end{array}$ \\
\hline 205822_s_at & 0.4701 & 0.0099 & HMGCS1 & $\begin{array}{l}\text { 3-hydroxy-3-methylglutaryl-Coenzyme } \\
\text { A synthase } 1 \text { (soluble) }\end{array}$ & hydroxymethylglutaryl-CoA synthase activity, transferase activity \\
\hline 211892_s_at & 0.2027 & 0.0004 & PTGIS & prostaglandin I2 (prostacyclin) synthase & $\begin{array}{l}\text { heme binding, iron ion binding, isomerase activity, metal ion } \\
\text { binding, monooxygenase activity, prostaglandin-I synthase activity }\end{array}$ \\
\hline 211019_s_at & 0.1844 & 0.0058 & LSS & $\begin{array}{l}\text { lanosterol synthase } \\
\text { (2;3-oxidosqualene-lanosterol cyclase) }\end{array}$ & $\begin{array}{l}\text { catalytic activity, isomerase activity, lanosterol synthase activity, } \\
\text { lyase activity }\end{array}$ \\
\hline
\end{tabular}

\#: Meta., distal metastasis developed during follow-up; Non-Meta.: no metastasis developed. 


\section{Patient characteristics and HSD17B2 expression in upper urinary tract urothelial carcinoma}

Table 2 shows the correlations between HSD17B2 expression and clinicopathological variables. Among the 340 patients with upper urinary tract urothelial carcinoma, 182 patients were female, and 202 patients were older than 65 years old at the time of diagnosis. The most common tumor location was the ureter, followed by the renal pelvis and both the renal pelvis and the ureter. Most upper urinary tract urothelial carcinomas were present in one location in the urinary system rather than as multifocal tumors. In these patients, noninvasive papillary urothelial carcinoma (Ta) was diagnosed in
$89(26 \%)$ patients, $92(27 \%)$ patients had tumors that had invaded the subepithelial connective tissue (T1), and $159(47 \%)$ patients had a tumor with a stage higher than T2. Most patients with upper tract urothelial carcinoma had no nodal metastasis, no vascular invasion, and no perineural invasion, and approximately $51 \%$ of the tumors displayed a low mitotic rate. In this study, we found that HSD17B2 expression was not associated with gender, age, tumor location, multifocality, or mitotic rate. A high level of expression of HSD17B2 was correlated with tumor stage in Ta and T1, less nodal metastasis, less vascular invasion, less perineal invasion and a lower histological grade (Figure 2).

Table 2. Correlations between RNF128 Expression and other important clinicopathological parameters in urothelial carcinomas.

\begin{tabular}{|c|c|c|c|c|c|c|c|c|c|}
\hline \multirow[t]{3}{*}{ Parameter } & \multirow[t]{3}{*}{ Category } & \multicolumn{4}{|c|}{ Upper Urinary Tract Urothelial Carcinoma } & \multicolumn{4}{|c|}{ Urinary Bladder Urothelial Carcinoma } \\
\hline & & \multirow[t]{2}{*}{ Case No. } & \multicolumn{2}{|c|}{ HSD17B2 Expression } & \multirow[t]{2}{*}{ p-value } & \multirow[t]{2}{*}{ Case No. } & \multicolumn{2}{|c|}{ HSD17B2 Expression } & \multirow[t]{2}{*}{ p-value } \\
\hline & & & High & Low & & & High & Low & \\
\hline \multirow[t]{2}{*}{ Gender } & Male & 158 & 79 & 79 & 1.000 & 216 & 105 & 111 & 0.489 \\
\hline & Female & 182 & 91 & 91 & & 79 & 42 & 37 & \\
\hline \multirow[t]{2}{*}{ Age (years) } & $<65$ & 138 & 69 & 69 & 1.000 & 121 & 67 & 54 & 0.112 \\
\hline & $\geq 65$ & 202 & 101 & 101 & & 174 & 80 & 94 & \\
\hline \multirow[t]{3}{*}{ Tumor location } & Renal pelvis & 141 & 61 & 80 & 0.093 & - & - & - & - \\
\hline & Ureter & 150 & 84 & 66 & & - & - & - & - \\
\hline & Renal pelvis \& ureter & 49 & 25 & 24 & & - & - & - & - \\
\hline \multirow[t]{2}{*}{ Multifocality } & Single & 278 & 138 & 140 & 0.779 & - & - & - & - \\
\hline & Multifocal & 62 & 32 & 30 & & - & - & - & - \\
\hline \multirow[t]{3}{*}{ Primary tumor $(\mathrm{T})$} & Ta & 89 & 59 & 30 & $<0.001^{*}$ & 84 & 52 & 32 & $<0.001^{*}$ \\
\hline & $\mathrm{T} 1$ & 92 & 63 & 29 & & 88 & 52 & 36 & \\
\hline & $\mathrm{T} 2-\mathrm{T} 4$ & 159 & 48 & 111 & & 123 & 43 & 80 & \\
\hline \multirow[t]{2}{*}{ Nodal metastasis } & Negative (N0) & 312 & 163 & 149 & $0.006^{*}$ & 266 & 141 & 125 & $0.001^{*}$ \\
\hline & Positive (N1-N3) & 28 & 7 & 21 & & 29 & 6 & 23 & \\
\hline \multirow[t]{2}{*}{ Histological grade } & Low grade & 56 & 60 & 38 & $0.008^{*}$ & 56 & 31 & 25 & 0.358 \\
\hline & High grade & 284 & 110 & 132 & & 239 & 116 & 123 & \\
\hline \multirow[t]{2}{*}{ Vascular invasion } & Absent & 234 & 141 & 93 & $<0.001^{*}$ & 246 & 128 & 118 & 0.090 \\
\hline & Present & 106 & 29 & 77 & & 49 & 19 & 30 & \\
\hline \multirow[t]{2}{*}{ Perineural invasion } & Absent & 321 & 167 & 154 & $0.002^{*}$ & 275 & 141 & 134 & 0.066 \\
\hline & Present & 19 & 3 & 16 & & 20 & 6 & 14 & \\
\hline \multirow{2}{*}{$\begin{array}{l}\text { Mitotic rate (per } 10 \\
\text { high-power fields) }\end{array}$} & $<10$ & 173 & 94 & 79 & 0.104 & 139 & 76 & 63 & 0.116 \\
\hline & $>=10$ & 167 & 76 & 91 & & 156 & 71 & 85 & \\
\hline
\end{tabular}

* Statistically significant.
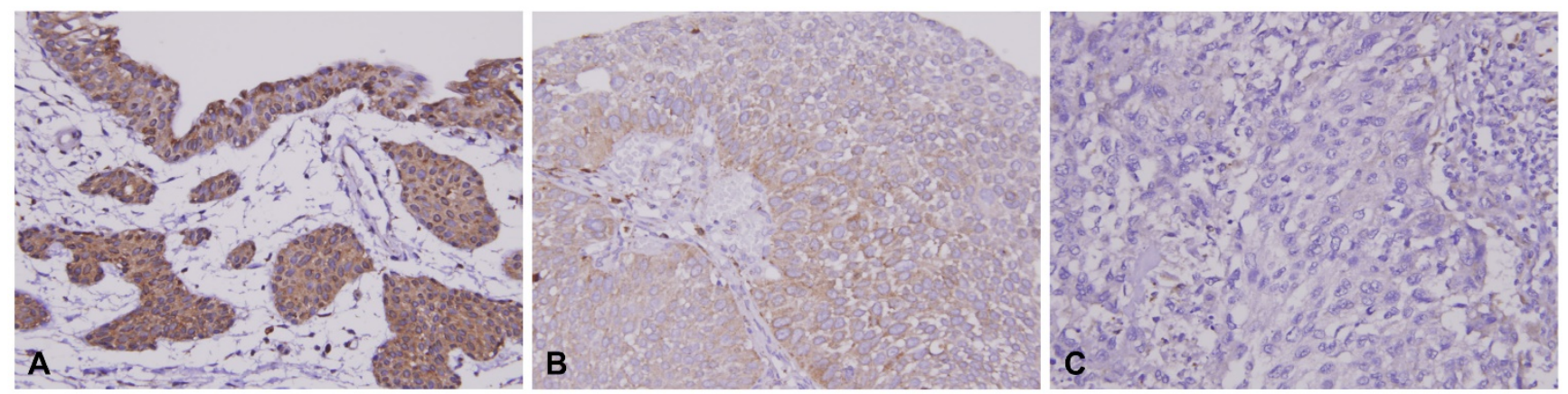

Figure 2. HSD17B2 immunostaining of representative tissue sections revealed a progressive stepwise decrease in the expression of HSD17B2 in non-tumoral urothelium and in (A) non-invasive (B) and infiltrating urothelial carcinoma (C) (X400). 


\section{Patient characteristics and HSD17B2 expression in urinary bladder urothelial carcinoma}

There were 295 patients with urinary bladder urothelial carcinoma in the study population. In this group, $216(73 \%)$ patients were male, and 174 (67\%) patients were older than 65 years old at the time of diagnosis. There were $84(28 \%)$ papillary noninvasive carcinomas (Ta), 88 (30\%) patients in whom the tumor had invaded the subepithelial connective tissue (T1), and $123(57 \%)$ patients with tumors that were staged higher than T2. Most patients with urinary bladder urothelial carcinoma had no nodal metastasis, no vascular invasion, no perineural invasion, and a low rate of mitotic activity. In urinary bladder, urothelial carcinomas, we found that HSD17B2 expression was not associated with gender, age, histological grade, vascular invasion, perineural invasion or mitotic rate. In contrast to upper urinary tract urothelial carcinoma, in urinary bladder urothelial carcinoma, a high level of expression of HSD17B2 was correlated only with $\mathrm{Ta}$ and $\mathrm{T} 1$ tumor stages and less nodal metastasis.

\section{Prognosis in upper urinary tract urothelial carcinoma}

Table 3 shows the results of the univariate and multivariate analyses for disease-specific and metastasis-free survival in upper urinary tract urothelial carcinoma. In the univariate analyses for disease-specific survival, the statistically significant parameters included tumor location, multifocality, primary tumor, nodal metastasis, histological grade, vascular invasion, perineal invasion and HSD17B2 expression. After multivariate analysis, only nodal metastasis, histological grade, perineural invasion and HSD17B2 expression were significantly associated with disease-specific survival. The multivariate analysis of metastasis-free survival revealed associations with multifocality, nodal metastasis, histological grade, and HSD17B2 expression. Moreover, of the clinicopathological parameters that affected outcomes in urothelial carcinoma, a high expression level of HSD17B2 was associated with better outcomes in upper urinary tract urothelial carcinoma.

Table 3. Univariate log-rank and multivariate analyses of Disease-specific and Metastasis-free Survival in upper urinary tract urothelial carcinoma.

\begin{tabular}{|c|c|c|c|c|c|c|c|c|c|c|c|c|}
\hline \multirow[t]{3}{*}{ Parameter } & \multirow[t]{3}{*}{ Category } & \multirow{3}{*}{$\begin{array}{l}\text { Case } \\
\text { No. }\end{array}$} & \multicolumn{5}{|c|}{ Disease-specific Survival } & \multicolumn{5}{|c|}{ Metastasis-free Survival } \\
\hline & & & \multicolumn{2}{|c|}{ Univariate analysis } & \multicolumn{3}{|c|}{ Multivariate analysis } & \multicolumn{2}{|c|}{ Univariate analysis } & \multicolumn{3}{|c|}{ Multivariate analysis } \\
\hline & & & No. of event & p-value & R.R. & 95\% C.I. & p-value & No. of event & p-value & R.R. & 95\% C.I. & p-value \\
\hline \multirow[t]{2}{*}{ Gender } & Male & 158 & 28 & 0.8286 & - & - & - & 32 & 0.7904 & - & - & - \\
\hline & Female & 182 & 33 & & - & - & - & 38 & & - & - & - \\
\hline \multirow[t]{2}{*}{ Age (years) } & $<65$ & 138 & 26 & 0.9943 & - & - & - & 30 & 0.8470 & - & - & - \\
\hline & $\geq 65$ & 202 & 35 & & - & - & - & 40 & & - & - & - \\
\hline \multirow[t]{3}{*}{ Tumor side } & Right & 177 & 34 & 0.7366 & - & - & - & 38 & 0.3074 & - & - & - \\
\hline & Left & 154 & 26 & & - & - & - & 32 & & - & - & - \\
\hline & Bilateral & 9 & 1 & & - & - & - & 0 & & - & - & - \\
\hline \multirow[t]{3}{*}{ Tumor location } & Renal pelvis & 141 & 24 & $0.0079^{*}$ & 1 & - & 0.972 & 31 & 0.0659 & - & - & - \\
\hline & Ureter & 150 & 22 & & 0.990 & $0.273-3.587$ & & 25 & & - & - & - \\
\hline & $\begin{array}{l}\text { Renal pelvis \& } \\
\text { ureter }\end{array}$ & 49 & 15 & & 0.917 & $0.226-3.732$ & & 14 & & - & - & - \\
\hline \multirow[t]{2}{*}{ Multifocality } & Single & 273 & 48 & $0.0026^{*}$ & 1 & - & 0.107 & 52 & $0.0127^{*}$ & 1 & - & $0.002^{*}$ \\
\hline & Multifocal & 62 & 18 & & 2.806 & $0.800-9.840$ & & 18 & & 2.372 & $1.367-4.117$ & \\
\hline \multirow[t]{3}{*}{ Primary tumor $(\mathrm{T})$} & $\mathrm{Ta}$ & 89 & 2 & $<0.0001^{*}$ & 1 & - & 0.151 & 4 & $<0.0001^{*}$ & 1 & - & 0.090 \\
\hline & $\mathrm{T} 1$ & 92 & 9 & & 4.191 & $0.885-19.853$ & & 15 & & 3.401 & $1.107-10.456$ & \\
\hline & $\mathrm{T} 2-\mathrm{T} 4$ & 159 & 50 & & 4.376 & $0.963-19.898$ & & 51 & & 2.312 & $0.727-7.350$ & \\
\hline \multirow[t]{2}{*}{ Nodal metastasis } & Negative (N0) & 312 & 42 & $<0.0001^{*}$ & 1 & - & $<0.001^{*}$ & 55 & $<0.0001^{*}$ & 1 & - & $<0.001^{*}$ \\
\hline & Positive (N1-N3) & 28 & 19 & & 6.094 & $3.285-11.306$ & & 15 & & 3.309 & $1.778-6.155$ & \\
\hline \multirow[t]{2}{*}{ Histological grade } & Low grade & 56 & 4 & $0.0215^{*}$ & 1 & - & $0.004^{*}$ & 3 & $0.0027^{*}$ & 1 & - & $0.036^{*}$ \\
\hline & High grade & 284 & 57 & & 4.337 & $1.597-11.776$ & & 67 & & 2.326 & $1.055-5.128$ & \\
\hline \multirow[t]{2}{*}{ Vascular invasion } & Absent & 234 & 24 & $<0.0001^{*}$ & 1 & - & 0.148 & 26 & $<0.0001^{*}$ & 1 & - & $0.004^{*}$ \\
\hline & Present & 106 & 37 & & 1.568 & $0.853-2.881$ & & 44 & & 2.395 & $1.325-4.329$ & \\
\hline \multirow[t]{2}{*}{ Perineural invasion } & Absent & 321 & 50 & $<0.0001^{*}$ & 1 & - & $0.001^{*}$ & 61 & $<0.0001^{*}$ & 1 & - & $0.027^{*}$ \\
\hline & Present & 19 & 11 & & 3.625 & $1.723-7.626$ & & 9 & & 2.312 & $1.101-4.854$ & \\
\hline \multirow{2}{*}{$\begin{array}{l}\text { Mitotic rate (per } 10 \\
\text { high-power fields) }\end{array}$} & $<10$ & 173 & 27 & 0.167 & - & - & & 30 & 0.0823 & - & - & \\
\hline & $>=10$ & 167 & 34 & & - & - & & 40 & & - & - & \\
\hline \multirow{2}{*}{$\begin{array}{l}\text { HSD17B2 } \\
\text { expression }\end{array}$} & High & 170 & 15 & $<0.0001^{*}$ & 1 & - & $0.031^{*}$ & 17 & $<0.0001^{*}$ & 1 & - & $0.003^{*}$ \\
\hline & Low & 170 & 46 & & 2.027 & $1.066-3.856$ & & 53 & & 2.438 & $1.2352-4.395$ & \\
\hline
\end{tabular}

* Statistically significant. 


\section{Prognosis in urinary bladder urothelial carcinoma}

Table 4 shows the results of the univariate and multivariate analyses used to analyze associations with disease-specific and metastasis-free survival in urinary bladder urothelial carcinoma. In the univariate analyses, many parameters, including primary tumor $(\mathrm{T})$, nodal metastasis, histological grade, vascular invasion, perineal invasion, mitotic rate and HSD17B2 expression, had significant prognostic value for predicting both disease-specific survival and metastasis-free survival. After the multivariate analysis, only primary tumor $(\mathrm{T})$, mitotic rate and HSD17B2 expression were significantly associated with prognosis.

In addition to these clinicopathological parameters, a high level of HSD17B2 expression was associated with better outcomes in urinary bladder urothelial carcinoma. In Figure 3, the results of log-rank tests show that that a high level of expression of HSD17B2 was a novel predictive marker of a better prognosis, including disease-specific survival and metastasis-free survival, in urothelial carcinoma of the urinary system.

\section{Discussion}

Evaluating the level of expression of HSD17B2 could significantly impact outcome predictions in urinary tract urothelial carcinoma. This is the first study to explore the relationship between HSD17B2 and urothelial carcinoma of the urinary tract system. Although adjuvant chemotherapy provides an overall survival benefit in patients with macroscopic perivesicular tissue invasion [12], the prognosis for these patients when they have urinary tract urothelial carcinoma remains poor [13]. A previous study focused on identifying markers of poor prognosis. Ma et al. [ ${ }^{7]}$ showed that a higher level of expression of SPOCK1 was associated with adverse outcomes in urothelial carcinoma. Chang et al. [8] found that a member of the melanoma-associated antigen gene (MAGE) family, NDN, predicted a poor prognosis in patients with urothelial carcinomas. Lee at al. [9] showed that overexpressing colony-stimulating factor 2 advanced the disease status and was associated with a poor prognosis in patients with urothelial carcinoma, suggesting that colony-stimulating factor 2 may be an important prognostic indicator and a potential therapeutic target. Our study shows that HSD17B2 expression is positively correlated with prognosis in urothelial carcinoma of the urinary tract system.

Table 4. Univariate log-rank and multivariate analyses of Disease-specific and Metastasis-free Survival in urinary bladder urothelial carcinoma.

\begin{tabular}{|c|c|c|c|c|c|c|c|c|c|c|c|c|}
\hline \multirow[t]{3}{*}{ Parameter } & \multirow[t]{3}{*}{ Category } & \multirow{3}{*}{$\begin{array}{l}\text { Case } \\
\text { No. }\end{array}$} & \multicolumn{5}{|c|}{ Disease-specific Survival } & \multicolumn{5}{|c|}{ Metastasis-free Survival } \\
\hline & & & \multicolumn{2}{|c|}{ Univariate analysis } & \multicolumn{3}{|c|}{ Multivariate analysis } & \multicolumn{2}{|c|}{ Univariate analysis } & \multicolumn{3}{|c|}{ Multivariate analysis } \\
\hline & & & $\begin{array}{l}\text { No. of } \\
\text { event }\end{array}$ & p-value & R.R. & 95\% C.I. & p-value & $\begin{array}{l}\text { No. of } \\
\text { event }\end{array}$ & p-value & R.R. & 95\% C.I. & p-value \\
\hline \multirow[t]{2}{*}{ Gender } & Male & 216 & 41 & 0.4446 & - & - & - & 60 & 0.2720 & - & - & - \\
\hline & Female & 79 & 11 & & - & - & - & 16 & & - & - & - \\
\hline \multirow[t]{2}{*}{ Age (years) } & $<65$ & 121 & 17 & 0.1136 & - & - & - & 31 & 0.6875 & - & - & - \\
\hline & $\geq 65$ & 174 & 35 & & - & - & - & 45 & & - & - & - \\
\hline \multirow[t]{3}{*}{ Primary tumor $(\mathrm{T})$} & $\mathrm{Ta}$ & 84 & 1 & $<0.0001^{*}$ & 1 & - & $0.001^{*}$ & 4 & $<0.0001^{*}$ & 1 & - & $0.013^{*}$ \\
\hline & $\mathrm{T} 1$ & 88 & 9 & & 6.498 & $0.670-63.037$ & & 23 & & 5.134 & $1.460-18.051$ & \\
\hline & $\mathrm{T} 2-\mathrm{T} 4$ & 123 & 42 & & 23.551 & 2.454-226.044 & & 49 & & 6.852 & $1.905-24.648$ & \\
\hline \multirow[t]{2}{*}{ Nodal metastasis } & Negative (N0) & 266 & 41 & $0.0002 *$ & 1 & - & 0.971 & 61 & $<0.0001^{*}$ & 1 & - & 0.239 \\
\hline & Positive (N1-N3) & 29 & 11 & & 0.987 & $0.483-2.018$ & & 15 & & 1.462 & $0.777-2.750$ & \\
\hline \multirow[t]{2}{*}{ Histological grade } & Low grade & 56 & 2 & $0.0013^{*}$ & 1 & - & 0.856 & 5 & $0.0007^{*}$ & 1 & - & 0.805 \\
\hline & High grade & 239 & 50 & & 0.860 & $0.169-4.368$ & & 71 & & 0.870 & $0.287-2.639$ & \\
\hline \multirow[t]{2}{*}{ Vascular invasion } & Absent & 246 & 37 & $0.0024^{*}$ & 1 & - & 0.415 & 54 & $0.0001^{*}$ & 1 & - & 0.449 \\
\hline & Present & 49 & 15 & & 0.748 & $0.372-1.503$ & & 22 & & 1.269 & $0.686-2.347$ & \\
\hline \multirow[t]{2}{*}{ Perineural invasion } & Absent & 275 & 44 & $0.0001 *$ & 1 & - & 0.067 & 66 & $0.0007^{*}$ & 1 & - & 0.326 \\
\hline & Present & 20 & 8 & & 2.165 & $0.948-4.944$ & & 10 & & 1.442 & $0.694-2.994$ & \\
\hline \multirow{2}{*}{$\begin{array}{l}\text { Mitotic rate (per } 10 \\
\text { high-power fields) }\end{array}$} & $<10$ & 139 & 12 & $<0.0001^{*}$ & 1 & - & $0.023^{*}$ & 23 & $<0.0001^{*}$ & 1 & - & $0.032^{*}$ \\
\hline & $>=10$ & 156 & 40 & & 2.169 & $1.113-4.229$ & & 53 & & 1.756 & $1.050-2.36$ & \\
\hline \multirow{2}{*}{$\begin{array}{l}\text { HSD17B2 } \\
\text { expression }\end{array}$} & High & 147 & 11 & $<0.0001^{*}$ & 1 & - & $0.001^{*}$ & 22 & $<0.0001^{*}$ & 1 & - & $<0.001^{*}$ \\
\hline & Low & 148 & 41 & & 3.288 & $1.630-6.634$ & & 54 & & 2.689 & $1.585-4.562$ & \\
\hline
\end{tabular}



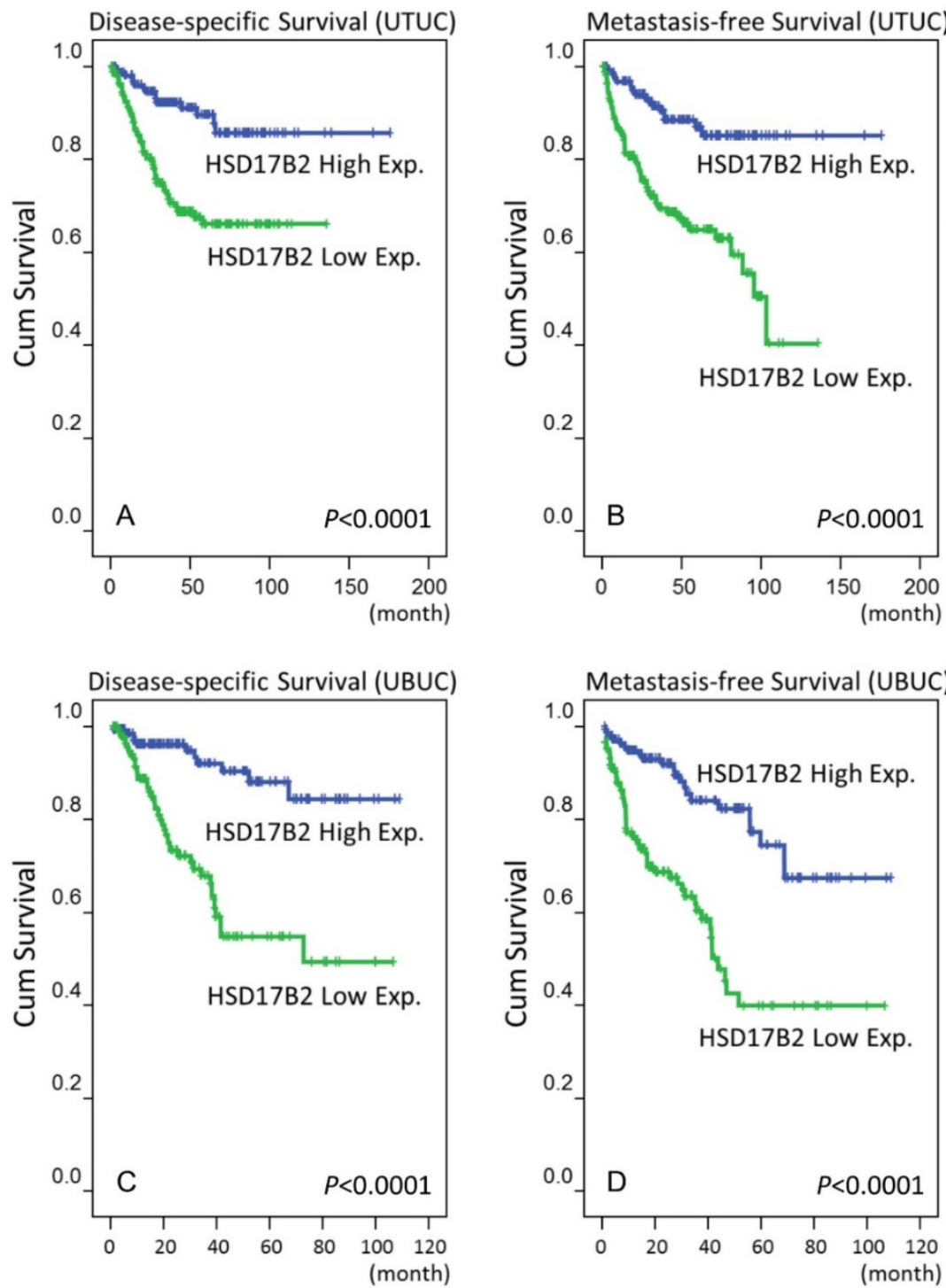

Figure 3. A survival analysis was plotted using Kaplan-Meier curves. Using a log-rank test, we found that a high level of HSD 17B2 expression predicted favorable disease-specific survival in urothelial carcinoma of the upper tract $(A)$ and urinary bladder $(C)$ and favorable metastasis-free survival in urothelial carcinoma of the upper tract (B) and urinary bladder (D).

HSD17B2 protects tissues from excessive steroid activity and is abundantly expressed in a variety of human tissues, such as the placenta, uterus, and liver and the gastrointestinal and urinary tracts [14]. In one study of cancer cell lines, while $17 \beta-H y d r o x y s t e r o i d$ dehydrogenases type 1 expression was higher in T47D breast cancer and JEG3 choriocarcinoma cells, HSD17B2 activity was low in both of these lines [15].

A previous study investigated the role of HSD17B2 in colon cancer and found that the expression of HSD17B2 was downregulated in colon cancer. A higher percentage of female patients with colon cancer than male patients with colon cancer overexpressed HSD17B2. Additionally, the 5-year overall survival rate in female patients with low expression levels of HSD17B2 was 54\%, and none of the female patients with HSD17B2 overexpression survived. The authors suggested that the HSD17B2 mRNA is an independent marker of a poor prognosis in female patients with colon cancer, but there was no association between HSD17B2 and prognosis in male patients with colon cancer [16]. In contrast to colon cancer, our study demonstrated that a high level of expression of HSD17B2 is a favorable prognostic indicator in urothelial carcinoma and that this relationship was not associated with gender. It is important to identify the different and sophisticated regulatory mechanisms that are affected by HSD17B2 to drive tumorigenesis in different types of tumors.

Previous studies have shown that HSD17B2 has been detected in both benign and malignant ovarian tumor tissues, but its activity level varies between samples [17-19]. In cell line studies, both the enzymatic activity and mRNA expression of 
HSD17B2 have been observed in epithelial cancer cells. HSD17B2 has both anti-estrogenic and anti-androgenic functions. Because different receptors for estrogen, androgen and progesterone have been detected in ovarian epithelial tumors, the role of HSD17B2 in the tumorigenesis of ovarian cancer remains controversial [20].

HSD17B2 can convert estradiol to the less active estrone. In normal breast tissue, the oxidative pathway (from estradiol to estrone) predominates in epithelial and stromal cells. However, in breast cancer cells, the reductive pathway is dominant [21]. For breast cancer cells to maintain an appropriate concentration of estradiol, the expression of HSD17B2 must therefore be reduced. However, the expression of $17 \beta$-hydroxysteroid dehydrogenase type 12 was found to be high [22]. In that study, because only a small number of cases had breast cancer, no significant association was found between HSD17B2 expression and other clinicopathological parameters [19]. Another study demonstrated that a high level of expression of HSD17B2 decreased the risk of later recurrence in patients with estrogen receptor-positive breast cancer [23]. In addition to estrogen receptor-positive breast cancer, our study also revealed a trend toward improved overall survival in urothelial carcinoma patients with high expression levels of HSD17B2.

Steroid hormones, including HSD17B2, play important roles in the development of breast cancer [22], ovarian cancer [18], endometrial cancer and prostate cancer [24]. The distribution and function of steroid hormone receptors in these tumor cells may have potential effects on tumor progression. The relationships between steroid hormone expression and the clinical stages of urothelial carcinoma of the urinary tract system remain unclear. Our results suggest that HSD17B2 is involved in the regulation of hormone balance in urothelial carcinoma of the urinary tract system and that this factor has a substantial impact on tumor growth and development. We propose that not only HSD17B2, but many other enzymes that are required for steroid biosynthesis and metabolism and therefore crucial for tumor growth, could be inhibited to explore their therapeutic potential.

In conclusion, our study revealed that HSD17B2 plays an important role in urothelial carcinoma of the urinary tract. High expression of HSD17B2 was associated with better clinicopathological parameters and was also an independent prognostic indicator. Hence, future studies aimed at determining the regulatory mechanism underlying the activity of HSD17B2 in urothelial carcinoma and developing this marker as a potential novel therapeutic target for cancer treatment are warranted.

\section{Acknowledgements}

This work is supported by grants from the Chi Mei Medical Center. This study was also supported by grants from Kaohsiung Medical University "Aim for the Top Universities" (KMU-TP104E31, KMU-TP105G00, KMU-TP105G01, KMU-TP105G02), the health and welfare surcharge of tobacco products, Ministry of Health and Welfare (MOHW105-TDU-B-212-134007), Ministry of Science and Technology (MOST103-2314-B-037-059-MY3, MOST 104-2314-B-037-050-MY3), and Kaohsiung Medical University Hospital (KMUH101-1R46, KMUH103-3M37).

\section{Ethical Standard}

This study was approved by the Institutional Review Board (IRB) of Chi Mei Medical Center (IRB10501005). All samples were obtained from the BioBank of Chi Mei Medical Center and had been previously collected according to official ethical guidelines. Informed consent was obtained from all patients whose tissues were enrolled into the BioBank.

\section{Supplementary Material}

Supplementary table S1 and figure S1.

http://www.jcancer.org/v07p2221s1.pdf

\section{Competing Interests}

The authors have declared that no competing interest exists.

\section{References}

1. Bulun SE, Cheng YH, Pavone ME, et al. $17 \beta$-Hydroxysteroid dehydrogenase-2 deficiency and progesterone resistance in endometriosis. Semin Reprod Med. 2010; 28: 44-50.

2. Moeller G, Adamski J. Integrated view on 17beta-hydroxysteroid dehydrogenases. Mol Cell Endocrinol. 2009; 301: 7-19.

3. Wu L, Einstein M, Geissler WM, et al. Expression cloning and characterization of human 17 beta-hydroxysteroid dehydrogenase type 2, a microsomal enzyme possessing 20 alpha-hydroxysteroid dehydrogenase activity. J Biol Chem. 1993; 268: 12964-9.

4. Green VL, Speirs V, Landolt AM, et al. 17Beta-hydroxysteroid dehydrogenase type 1, 2, 3, and 4 expression and enzyme activity in human anterior pituitary adenomas. J Clin Endocrinol Metab. 1999; 84: 1340-5.

5. Jansson A, Carlsson J, Olsson A, et al. A new polymorphism in the coding region of exon four in HSD17B2 in relation to risk of sporadic and hereditary breast cancer. Breast Cancer Res Treat. 2007; 106: 57-64.

6. Cunningham JM, Hebbring SJ, McDonnell SK, et al. Evaluation of genetic variations in the androgen and estrogen metabolic pathways as risk factors for sporadic and familial prostate cancer. Cancer Epidemiol Biomarkers Prev. 2007; 16: 969-78

7. Ma LJ, Wu WJ, Wang YH, et al. SPOCK1 Overexpression confers a poor prognosis in urothelial carcinoma. J Cancer. 2016; 7: 467-76.

8. Chang IW, Wang $\mathrm{YH}, \mathrm{Wu}$ WJ, et al. Necdin overexpression predicts poor prognosis in patients with urothelial carcinomas of the upper urinary tract and urinary bladder. J Cancer. 2016; 7: 304-13.

9. Lee $Y Y, W u$ WJ, Huang CN, et al. CSF2 Overexpression is associated with STAT5 phosphorylation and poor prognosis in patients with urothelial carcinoma. J Cancer. 2016; 7: 711-21.

10. Liao KM, Chao TB, Tian YF, et al. Overexpression of the PSAT1 gene in nasopharyngeal carcinoma is an indicator of poor prognosis. J Cancer. 2016; 7: 1088-94. 
11. Eble JN, World Health Organization, International Agency for Research on Cancer, et al. Pathology and genetics of tumours of the urinary system and male genital organs. World Health Organization classification of tumours. Lyon, France: IARC Press; 2004

12. Kim HS, Piao S, Moon KC, et al. Adjuvant chemotherapy correlates with improved survival after radical cystectomy in patients with pT3b (macroscopic perivesical tissue invasion) bladder cancer. J Cancer. 2015; 6: 750-8.

13. Tilki D, Svatek RS, Karakiewicz PI, et al. pT3 Substaging is a prognostic indicator for lymph node negative urothelial carcinoma of the bladder. J Urol. 2010; 184: 470-4.

14. Peltoketo $\mathrm{H}$, Luu-The V, Simard J, et al. 17beta-hydroxysteroid dehydrogenase (HSD)/17-ketosteroid reductase (KSR) family; nomenclature and main characteristics of the 17HSD/KSR enzymes. J Mol Endocrinol. 1999; 23: 1-11.

15. Day JM, Tutill HJ, Newman SP, et al. 17Beta-hydroxysteroid dehydrogenase Type 1 and Type 2: association between mRNA expression and activity in cell lines. Mol Cell Endocrinol. 2006; 248: 246-9.

16. Oduwole OO, Mäkinen MJ, Isomaa VV, et al. 17Beta-hydroxysteroid dehydrogenase type 2: independent prognostic significance and evidence of estrogen protection in female patients with colon cancer. J Steroid Biochem Mol Biol. 2003; 87: 133-40.

17. Blomquist $\mathrm{CH}$, Bonenfant M, McGinley DM, et al. Androgenic and estrogenic 17beta-hydroxysteroid dehydrogenase/17-ketosteroid reductase in human ovarian epithelial tumors: evidence for the type 1, 2 and 5 isoforms. J Steroid Biochem Mol Biol. 2002; 81: 343-51.

18. Chura JC, Ryu HS, Simard M, et al. Steroid-converting enzymes in human ovarian carcinomas. Mol Cell Endocrinol. 2009; 301: 51-8.

19. Vierikko P, Kauppila A, Vihko R. Cytosol and nuclear estrogen and progestin receptors and 17 beta-hydroxysteroid dehydrogenase activity in non-diseased tissue and in benign and malignant tumors of the human ovary. Int J Cancer. 1983; 32: 413-22.

20. Provost PR, Lima PH, Tremblay Y, et al. A useful cell system for studying the regulation of 17HSD/KSR type 2 activity and expression in ovarian epithelial cancer. J Steroid Biochem Mol Biol. 2010; 122: 295-301.

21. Speirs V, Green AR, Atkin SL. Activity and gene expression of 17beta-hydroxysteroid dehydrogenase type I in primary cultures of epithelial and stromal cells derived from normal and tumourous human breast tissue: the role of IL-8. J Steroid Biochem Mol Biol. 1998; 67: 267-74.

22. Han B, Li S, Song D, et al. Expression of 17beta-hydroxysteroid dehydrogenase type 2 and type 5 in breast cancer and adjacent non-malignant tissue: a correlation to clinicopathological parameters. J Steroid Biochem Mol Biol. 2008; $112: 194-200$

23. Gunnarsson C, Olsson BM, Stål O. Abnormal expression of 17beta-hydroxysteroid dehydrogenases in breast cancer predicts late recurrence. Cancer Res. 2001; 61: 8448-51.

24. Zakharov V, Lin HK, Azzarello J, et al. Suppressed expression of type 2 3alpha/type 5 17beta-hydroxysteroid dehydrogenase (AKR1C3) in endometrial hyperplasia and carcinoma. Int J Clin Exp Pathol. 2010; 3: 608-17. 